\title{
RHEOLOGICAL PROPERTIES OF MR FLUIDS RECOMMENDED FOR USE IN SHOCK ABSORBERS
}

\author{
Bogdan SAPIŃSKI*, Wojciech HORAK ${ }^{* *}$
}

\author{
"AGH University of Science and Technology, Faculty of Mechanical Engineering and Robotics, Department of Process Control, \\ Al. Mickiewicza 30, 30-059 Kraków, Poland \\ "*AGH University of Science and Technology Faculty of Mechanical Engineering and Robotics, Department of Machine Design and Technology, \\ Al. Mickiewicza 30, 30-059 Kraków, Poland \\ deep@agh.edu.pl, horak@agh.edu.pl
}

\begin{abstract}
The paper summarises the results of laboratory testing of rheological behaviour of (magnetorheological) MR fluids designed for use in shock absorber and vibration dampers. The experiments used a rotational rheometer with an extra chamber inside which a uniform magnetic field can be generated. Underlying the description of rheological properties of fluids is the Herschel-Bulkley's model of viscous-plastic substances. The aim of the experiment was to determine the shear stress, yield stress, the yield factor and the power-law exponent depending on the magnetic flux density, followed by the comparative study of rheological parameters of investigated fluids.
\end{abstract}

Key words: MR Fluids, Rheological Characteristics, Herschel-Bulkley's Model, Shear Stress, Yield Stress

\section{INTRODUCTION}

MR fluids are suspensions of ferromagnetic particles with the diameter 1-20 um in a carrier fluid which does not exhibit any magnetic properties. The fraction of ferromagnetic particles in a MR fluid ranges from 10 to $15 \%$ by volume. These particles are typically made of iron oxides or iron, nickel, cobalt alloys. Saturation magnetisation of their material is about 2.2T. The choice of the carrier fluid depends on the projected application requirements of MR fluids. Most widely used carrier fluids include mineral or synthetic oils, some of them are based on water, polyesters or polyeter (Cheng et al., 2009; Gorodkin et al., 2009; Jonsdottir et al., 2010; Phule, 2001). MR fluids may contain some additives to prevent sedimentation and aggregation of particles and to improve their lubrication properties. Some admixtures, mostly surfactants are added to improve the fluid stability. These include polymers or carboxyl acids (Du et al., 2010).

The unique feature of a MR fluid is that its rheological properties change under the action of the applied magnetic field. This change is instantaneous (the response time of MR fluids is of the order of several milliseconds) and reversible. Besides of their unique property, MR fluids are now widely used in devices with the controllable operating parameters, such as couplings, brakes, vibration dampers or shock absorbers (Gołdasz and Sapiński, 2011; Olabi and Grunwald, 2007; Sapiński, 2006). Presently, most widespread industrial applications of MR fluids include shock absorbers and vibration dampers. The structure and operating principles of MR shock absorbers is outlined in the comprehensive work (Zhu et al., 2012).

In the absence of magnetic field, MR fluid behaves like typical non-colloidal suspensions. Rheological properties of MR fluids in this state are dependent mostly on rheological parameters of the base fluid, properties of solid particles (size, shape and volumetric proportion).

Under the action of a magnetic field, the particles arrange themselves in spatial, columnar structures, along the magnetic field lines. In the macro-scale, that is observed as a change of rheological properties of the fluids. Through the application of magnetic field, the yield stress can be increased to $100 \mathrm{kPa}$ or even to $1400 \mathrm{kPa}$ providing the strain state of the fluid is modified accordingly (Olabi and Grunwald, 2007; Wang et al., 2011).

The MR effect, i.e. the change of rheological properties of the fluid under the action of magnetic field is associated mostly with magnetic properties of the material from which the particles are made. In the context of magnitude of MR effect, of particular importance is the saturation magnetisation of the particle material and the volumetric proportion of ferromagnetic particles in a MR fluid.

Rheological properties of a MR fluid are determined by several factors: rheological parameters of the base fluid, concentration and density of ferromagnetic particles, their dimensions, shape and magnetic properties. When analysing the behaviour of MR fluids, the effects of the presence of surfactants, thermal interactions and distribution of the applied magnetic field have to be taken into account.

Several MR fluids are now commercially available that are dedicated for use in vibration damping systems. Because of the variety of adopted measurement methods, the data provided in manufacturers' specifications may not allow a direct comparison between rheological properties of several fluids. The purpose of the present study is to obtain the characteristic of MR fluids and to compare the rheological properties of MR fluids recommended for use in shock absorbers.

\section{MR FLUIDS RECOMMENDED FOR USE IN SHOCK ABSORBERS}

Depending on their applications, commercially available MR fluids intended for use in system with controllable process parameters can be categorised into two groups: MR fluids for use in shock absorbers and those recommended for use in couplings and brakes. The main difference between their properties lies in the 
level of the achievable yield stress. For fluids designed for use in shock absorbers, the value of yield stress is less than $20 \mathrm{kPa}$, for fluids employed in couplings and brakes the yield stress can exceed $50 \mathrm{kPa}$. Apart from differences in composition of the two fluid types (addition of some improvers), the volumetric proportion of ferromagnetic particles will differ, too $(20 \%$ for MR fluids used in shock absorbers and over $40 \%$ in those applied in couplings and brakes).

Selection of a MR fluid to be used in a shock absorber should be optimised in terms of such parameters as: variability range of the yield stress under the action of magnetic field, stability of MR fluid (resistance to oxidation), resistance to sedimentation, ease of application, tribological properties and durability as well as the admissible temperature range for fluid operation. Another vital parameter of a MR fluid that impacts on the performance of shock absorbers is fluid viscosity in the absence of magnetic field.

The behaviour of MR fluids is typically governed by models of viscous-plastic substances, including the Bingham model, the bi-viscous model and the Herschel-Bulkley's model (Gołdasz, 2012). The first two models give an approximate description of the MR fluid behaviour whilst the Herschel-Bulkley's power law formula enables a more accurate representation of yield stress. The model can be expressed as:

$\tau=\tau_{0}+\eta \dot{\gamma}^{n}$

where: $\tau$ - shear stress, $\tau_{0}$ - yield stress, $\eta$ - visosity, $\dot{\gamma}$ - shear rate, $n$ - power-law exponent

Depending on the power law exponent, the model governs various aspects of fluid behaviour. For shear thinning substances $n<1$, whilst for shear thickening ones $n>1$. For $n=1$, this model becomes an equivalent of the Bingham model.

\section{EXPERIMENTS}

Testing is done on three MR fluids recommended for use in shock absorbers: MRF-122EG (Lord Corporation) two manufactured by the BASF company (Basonetic 2040, Basonetic 4035) (http://www.basonetic.com/, http://www.lord.com/). Similar density of tested fluids is indicative of similar proportion of solid particles. Selected properties of investigated fluids are compiled in Tab. 1.

Tab. 1. Properties of investigated MR fluids

\begin{tabular}{|l|c|c|}
\hline \multirow{2}{*}{ MR fluid } & Density & $\begin{array}{c}\text { Saturation } \\
\text { magnetization }\end{array}$ \\
\cline { 2 - 3 } & {$\left[\mathrm{g} / \mathrm{cm}^{3}\right]$} & {$[\mathrm{kA} / \mathrm{m}]$} \\
\hline Basonetic 2040 & 2.47 & $\sim 418$ \\
\hline Basonetic 4035 & 2.68 & $\sim 417$ \\
\hline MRF-122EG & 2.38 & $\sim 359$ \\
\hline
\end{tabular}

The experiments were performed using a rotational rheometer Physica MCR 301 (http://www.anton-paar.com/) complete with a test cell enabling the experiments to be conducted under a uniform magnetic field. The schematic diagram of the test cell is shown in Fig. 1.

A MR fluid sample (2) was placed between the electromagnet cores (4) and a rotational plate in a rheometer (1). The flux density was altered through the change of current intensity in the electromagnet windings (3). The closed chamber formed a magnetic circuit controlling the field distribution in such manner that field lines should be perpendicular to the shearing direction.

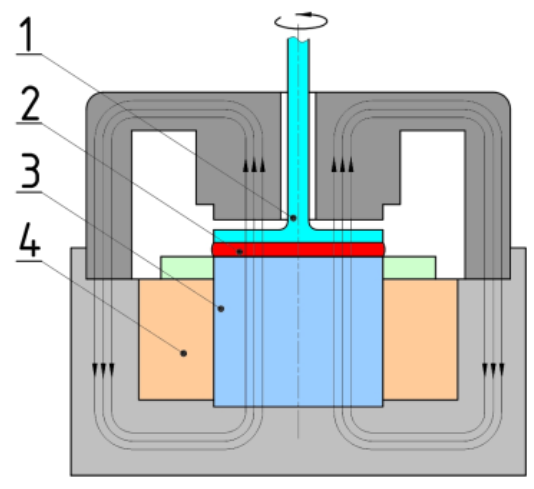

Fig. 1. Schematic diagram of the test cell

The testing was done in the experimental setup incorporating two parallel plates with the diameter $\varnothing 20 \mathrm{~mm}$, under the magnetic field with the flux density $B=0-0.5 T$ in the temperature-stabilised conditions (at the temperature $25^{\circ} \mathrm{C}$ ). The gap height was $\mathrm{h}=0.5$ $\mathrm{mm}$ and the volume of the tested fluid samples was $175 \mu$.

The experiments were conducted in two stages. In the first stage the shear stress curves were obtained under the linearly increasing flux density and for the constant shear rate 1001/s. In the second stage the yield curves were obtained for the constant flux density $\mathrm{B}: 0,0.1,0.2,0.3,0.4,0.5 \mathrm{~T}$. The results give us a better insight into rheological behaviour of investigated fluids.

Measurement data were then subjected to the correction procedure by the method suggested by Soskey and Winter (1984), on account of the non-Newtonian behaviour of MR fluids and the plate-plate configuration.

Fig. 2 shows the flow curves of investigated MR fluids, obtained in the absence of magnetic field.

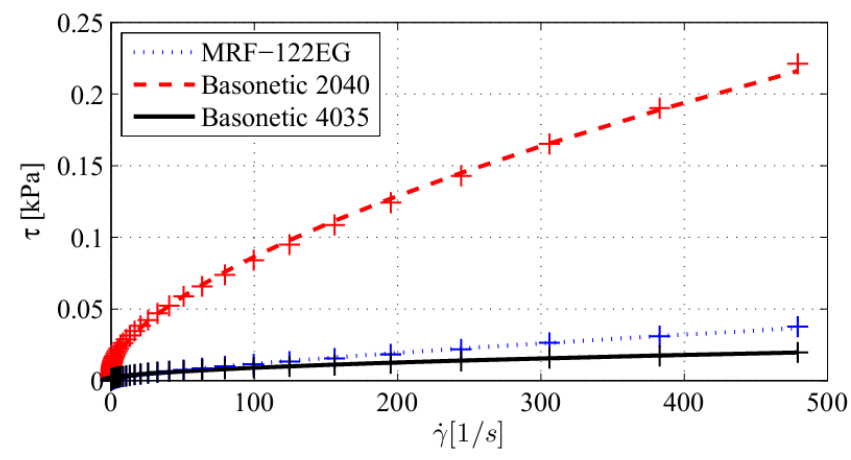

Fig. 2. Flow curves $(B=0 T)$

The values of shear stress obtained for Basonetic 4035 and MRF-122EG were relatively low, which suggests the low viscosity of the base fluid. In the case of Basonetic 2040, a yield stress limit is clearly revealed, which indicates it is based on a highviscosity oil.

The values of the Herschel-Bulkey's model parameters obtained for the investigated fluids in the absence of magnetic field are summarised in Tab. 2. Thus obtained yield stress plots are found to be non-linear.

Differences between the values of the $\mathrm{H}-\mathrm{B}$ exponent $\mathrm{n}$ may be indicative of the influence that the solid phase (ferromagnetic 
particles) has on the behaviour of the MR fluid in the absence of magnetic field. The values $n<1$ are characteristic of typical noncolloidal shear thinning suspensions.

Tab. 2. Parameters of the Herschel-Bulkey's model, $(B=0 T)$

\begin{tabular}{|l|c|c|c|}
\hline \multirow{2}{*}{ MR fluid } & Yield stress & Viscosity & H-B exponent \\
& $\tau_{0}$ & $\eta$ & $n$ \\
\cline { 2 - 4 } & {$[\mathrm{Pa}]$} & {$[\mathrm{Pa} \cdot \mathrm{s}]$} & {$[-]$} \\
\hline Basonetic 2040 & 6.45 & 4.7 & 0.61 \\
\hline Basonetic 4035 & 0.32 & 0.83 & 0.51 \\
\hline MRF-122EG & 0.48 & 0.37 & 0.74 \\
\hline
\end{tabular}

Fig. 3 shows the shear stress plots for investigated MR fluids under the linearly increasing magnetic flux density and for the fixed shear rate $(\dot{\gamma}=1001 / \mathrm{s})$. The range of shear stress values under the specified conditions was similar for all fluids. Differences in the stress values are observed when the flux density was $B \geq 0.2 T$.

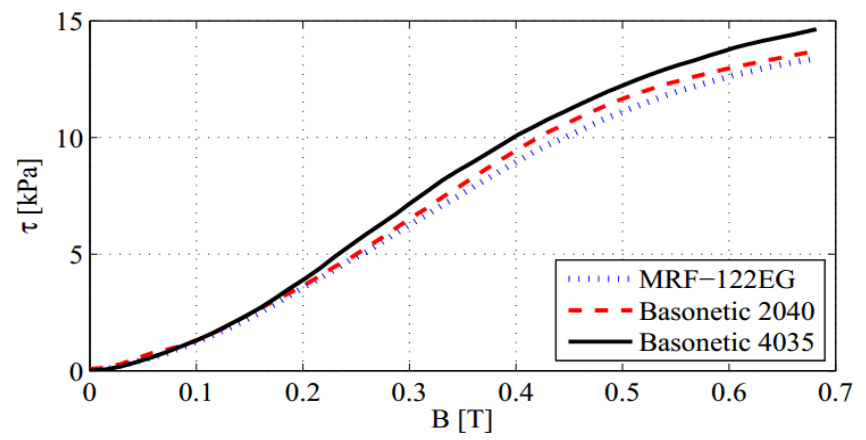

Fig. 3. Shear stress vs magnetic flux density $(\dot{\gamma}=1001 / \mathrm{s})$

The highest levels of the shear stress are registered for Basonetic 4035, which features low zero-state viscosity and high saturation magnetisation.

Fig. 4 shows the yield stress curves obtained by applying the Herschel-Bulkey's model to approximation of measurement points determined under variable flux densities.

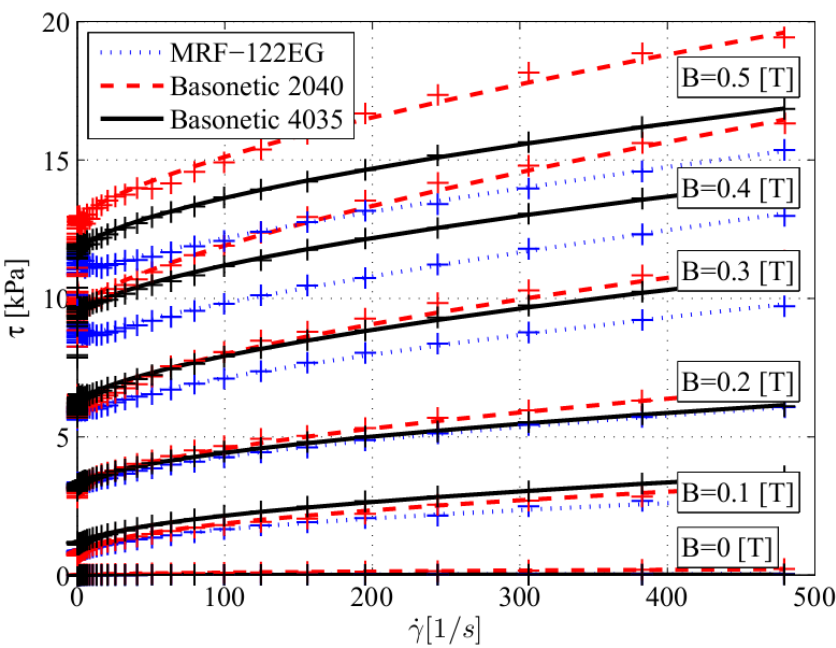

Fig. 4. Flow curves approximated by the Herschel-Bulkley's model

It appears that the adopted rheological model agrees well with the measurement data. Model coefficients used in further analyses are obtained by approximation.

The plots of yield stress obtained by the Herschel-Bulkey's model are shown in Fig. 5. At low levels of magnetic flux density, all fluids exhibit similar yield stress. More considerable differences between the values of this parameters for particular MR fluids are registered for $\mathrm{B}>0.3 \mathrm{~T}$. The lowest values of the yield stress are registered for the fluid with the lowest saturation magnetisation

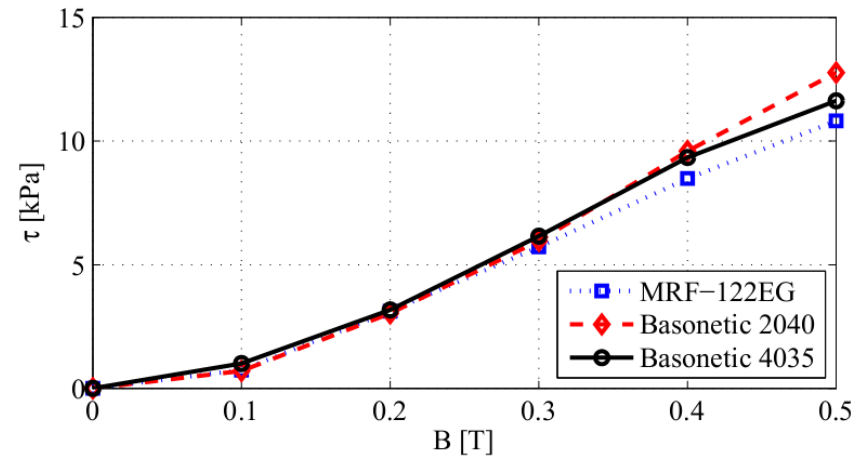

Fig. 5. Yield stress vs. magnetic flied density; Herschel-Bulkley's model

Fig. 6 shows the variability of the yield factor of the analysed rheological model.

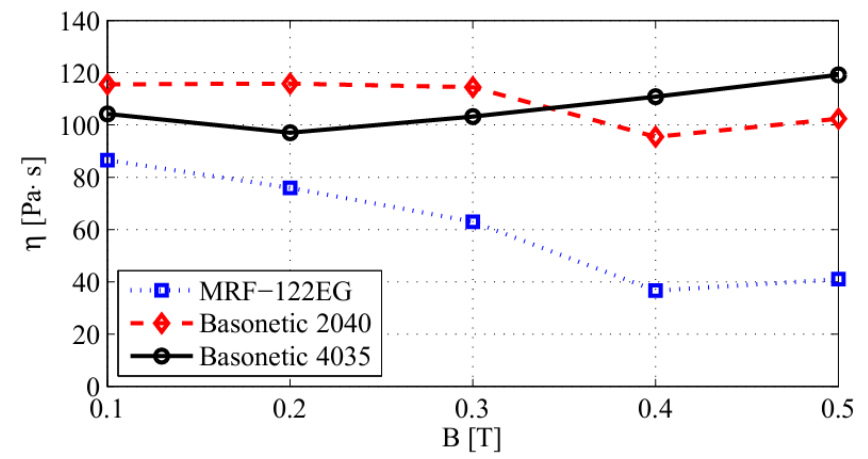

Fig. 6. Viscosity vs. magnetic flied density; Herschel-Bulkley's model

For MR fluids featuring similar magnetisation (Basonetic 2040, Basonetic 4035) in the investigated range of magnetic flux density, the values of the yield factor are similar. For the fluid having the lowest saturation magnetisation (MRF-122EG), the yield factor tends to decrease with an increased magnetic flux density.

Fig. 7 shows the plots of the exponent $n$ in HerschelBulkey's model (H-B exponent).

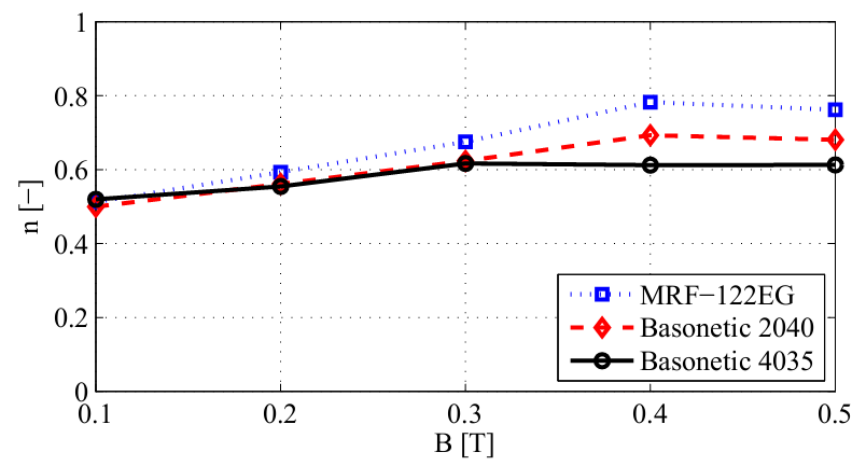

Fig. 7. Power-law exponent vs. magnetic flied density 
The rheological behaviour of investigated fluids is characteristic of shear thinning fluids $(n<1)$. It appears that the value of the exponent $\mathrm{n}$ tends to increase with an increase in the magnetic flux density. That indicates the lower level of shear thickening of investigated fluids under higher flux densities.

One has to bear in mind that in the model applied in Eq (1), the variability of the yield curve is associated with both the viscoisty $(\eta)$ and the power-law exponent $(n)$.

\section{SUMMARY}

W The paper summarises the results of laboratory testing of rheological behaviour of commercially available MR fluids (Basonetic 2040, Basonetic 4035, MRF-122EG), differing in their zero-state viscosity and in the level of saturation magnetisation.

Testing of rheological parameters of selected MR fluids indicates their similar behaviour. Plots of investigated parameters reveal that the considered range of magnetic flux density will not provide for saturation magnetisation of tested fluids. It is reasonable to expect a further increase of the yield limit, at higher levels of the magnetic flux density.

The upper limit of yield stress variability under the defined test conditions approaches $12 \mathrm{kPa}$.

Most significant differences between the behaviour of particular MR fluids are revealed in the absence of magnetic field and at high levels of magnetic flux density. At low flux densities, the differences between properties of MR fluids may be attributable to differences in their composition and rheological parameters of the base fluid. At higher values of magnetic flux density, the observed differences are mostly due to magnetic properties and differences in volumetric proportion of ferromagnetic particles in the MR fluids.

\section{REFERENCES}

1. Cheng H., Wang J., Zhang Q., Werely N. (2009), Preparation of composite magnetic particles and aqueous magnetorheological fluids, Smart Materials and Structures, 18, 8, 1-4.

2. Du C., Chen W., Wan F. (2010), Influence of HLB parameters of surfactants on properties of magnetorheological fluid, Advanced Materials Research, 97-101, 843-847.

3. Gołdasz J. (2012), Magnetorheological Shock Absorbers: Automotive Context, Wydawnictwo Politechniki Krakowskiej.

4. Gołdasz J., Sapiński B. (2011), Modeling of Magnetorheological Mounts in Various Operation Modes, Acta Mechanica and Automatica, Vol. 5, No. 4, 29-39.

5. Gorodkin S., James R., Kordonski W. (2009), Magnetic properties of carbonyl iron particles in magnetorheological fluids, Journal of Physics: Conference Series, 149(1), 1-4.

6. Jonsdottir F., Gudmundsson K. H., Dijkman T. B., Thorsteinsson F., Gutfleisch O. (2010), Rheology of perfluorinated polyether-based MR fluids with nanoparticles, Journal of Intelligent Material Systems and Structures, 21, 11, 1051-1060.

7. Olabi A., Grunwald A. (2007), Design and application of magnetorheological fluid, Materials and Design, 28(10), 2658-2664.

8. Phule P. (2001), Magnetorheological (MR) fluids: Principles and applications, Smart Materials Bulletin, 2001(2), 7-10.

9. Sapiński B. (2006), Magnetorheological dampers in vibration control, Uczelniane Wydawnictwa Naukowo-Dydaktyczne AGH, Kraków.

10. Soskey P. R,. Winter H. H. (1984), Large step shear strain experiments with parallel disk rotational rheometers, Journal of Rheology, 28, 625-645.

11. Wang H., Zhang B. J., Liu X. Z., Luo D. Z., Zhong S. B. (2011), Compression resistance of magnetorheological fluid, Advanced Materials Research, 143-144, 624-628.

12. Zhu X., Jing X., Li Ch. (2012), Magnetorheological fluid dampers: A review on structure design and analysis, Journal of Intelligent Material System and Structures, 23(8), 839-873.

13. Anton Paar, http://www.anton-paar.com/

14. BASF The Chemical Company, http://www.basonetic.com/

15. LORD Corporation, http://www.lord.com/

This research is supported by the National Centre for Research and Development under grant No. PBS 1/A6/3/2012. 